Ann. Biol. anim. Bioch. Biophys., I97I, 11 (I), II3-I27.

\title{
MODIFICATIONS APPORTÉES PAR LA CACUMECTOMIE A LA RÉPARTITION ET A LA NATURE DES CONSTITUANTS LIPIDIQUES DU CONTENU DIGESTIF DU LAPIN
}

\author{
Claude BACQUES et J.-P. PERRET \\ avec la collaboration technique de Dominique Richardot \\ Laboratoire de Physiologie, \\ 1 Institut universitaire de Technologie, \\ 43 Bd du 11 novembre, 69-Villeurbanne
}

\section{RÉSUMÉ}

Les données fragmentaires sur la digestion des lipides chez le Lapin nous ont conduit à l'élaboration d'un protocole de recherche dont la première étape a eu pour but de déterminer la répartition des lipides par segment intestinal et d'observer les modifications engendrées par la cæcumectomie.

Vingt lapins blancs placés en maintenance dans une batterie à distribution contrôlée d'aliments fourniront les prélèvements témoins. La cæcumectomie est pratiquée aseptiquement sur 5o lapins. cæcal.

Des techniques d'incubation in vitro et in vivo font apparaître le rôle lipolytique du contenu texte.

Les techniques d'analyse, adaptées au matériel digestif riche en eau, sont décrites dans le

Les résultats sont les suivants :

I $^{\circ}$ La courbe de croissance de l'opéré est comparable à celle du témoin. La survie se prolonge au-delà d'un an. Le phénomène de coprophagie disparaît. La consommation en granulés est plus faible.

$2^{\circ} \mathrm{Du}$ point de vue fonctionnel, on note :

a) un chyme digestif plus riche en eau chez l'animal opéré, eau qui sera absorbée par le côlon distal ce qui se traduira par l'émission de crottes normales ;

b) une élimination plus grande des graisses en valeur absolue, après intervention, sous la forme d'acides gras libres;

c) l'absence de saturation des acides en $\mathrm{C}_{18: 1,}{ }_{2}$ et 3 qui entrent dans l'estérification des stérols. L'hydrogénation est liée à la présence bactérienne.

La discussion débouche par des questions précises sur notre programme actuel de recherches faisant intervenir l'exclusion systématique de sécrétions ou de portions d'organes. 


\section{I. - IN'TRODUC'TION}

Nos connaissances sur le métabolisme lipidique des herbivores euthériens sont encore très limitées par rapport à ce que nous savons de celui des ruminants. En particulier, chez le lapin, cette étude n'a guère progressé depuis la mise en évidence de la production d'acides gras volatils (AGV) par fermentation cellulolytique dans le cæcum (BARCROFT et al., I944; Cools et JEUNIAUX, I96I). On trouvera dans la littérature, des renseignements sur le transit intestinal (KAMETAKA, I967) (YoshIHARA et KANDATSU, I960) en relation avec le péristaltisme (CoUL,LAUD et LívìquE, I966), sur l'absorption des lipides digestifs (LIPSKY et LANDOWNE, I963), ou sur le métabolisme particulier du cholestérol dans un but pharmacologique (POPJAK, I946, Bloom et al., r95I ; Cook et 'Thomson, I95I ; Cook et Thomson, I95I).

Aucune recherche systématique de la répartition des constituants lipidiques le long du tractus digestif n'a encore été entreprise. Nous n'avons également que très peu de données sur la flore et la faune cæcale dont on sait cependant qu'elle est susceptible de présenter à l'absorption une quantité importante d'aminoacides par lyse au niveau de l'estomac et du côlon proximal (VIaLIARD et RAYNAUD, I968), ce qui laisse supposer simultanément un apport substantiel de lipides. Enfin, nous savons que l'acide lactique représente l'essentiel de l'acidité organique du cæcum (BONNAFous et RAyNAUD, I968). Mais, et ceci est couramment admis (DUCLUZEAU, I969), nous n'avons pas mis en évidence de lactobacilles dans cet organe, ce qui rend d'autant plus difficile l'hypothèse d'une production in vivo d'acides gras longs (AGL) à partir d'acide lactique, par ce bacille, comme elle a été démontrée in vitro, à l'aide de corps marqués (SCHEUERBRANDT et BLOCK, I962; ERwiN et BLOCH, I964).

Quant aux expériences de cæcumectomie, elles sont réduites à celles de HERNDON et Hove (I955) dont les résultats nous semblent fort contestables.

Nous sommes donc amenés à traiter d'un problème nouveau pour lequel nous tenons à nous limiter à l'exposé des faits, nous réservant ultérieurement une étude plus approfondie des mécanismes.

Nous présenterons successivement les modifications anatomo-fonctionnelles apportées par la cæcumectomie, nos résultats d'analyses comparés entre lapin normal et opéré et les indications données par les techniques d'incubation in vitro et in vivo.

\section{II. - PROTOCOLE}

\section{I. - Épreuves sur lapins témoins}

Vingt lapins blancs de $2,500 \mathrm{~kg}$ provenant d'un élevage standardisé de Poitiers sont placés en batterie de cages individuelles à distribution d'eau automatique. Ils sont nourris exclusivement à l'aide de granulés industriels assurant un maximum d'humidité de $\mathrm{I}_{4} \mathrm{p}$. Ioo, de matière cellulosique de 5 p. Ioo et un minimum de matières minérales 9 p. Ioo, de matières protéiques brutes I 5 p. IOo, de matières grasses $3 \mathrm{p}$. Ioo.

Ils sont maintenus en stabulation pendant 3 semaines avant d'être utilisés pour l'expérimentation. On procède aux prélèvements de la façon suivante :

Les animaux à jeun depuis 12 heures sont anesthésiés par voie veineuse au pentobarbital sodique $\left(3^{\circ} \mathrm{mg} / \mathrm{kg}\right)$, puis sacrifiés par saignées simultanées à la carotide et à la veine porte. Le 
sang est recueilli sur héparine. Le tube intestinal est fractionné par simple pose de ligatures avant d'être extériorisé.

On sépare ainsi :

- le grêle en entier ;

- le cæcum que l'on divise en 5 portions.

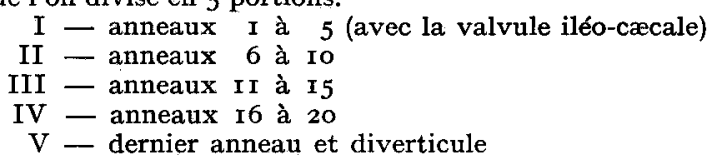

- côlon distal. diennes.

On prélève également un échantillon moyen de granulés par lot, ainsi que les crottes quoti-

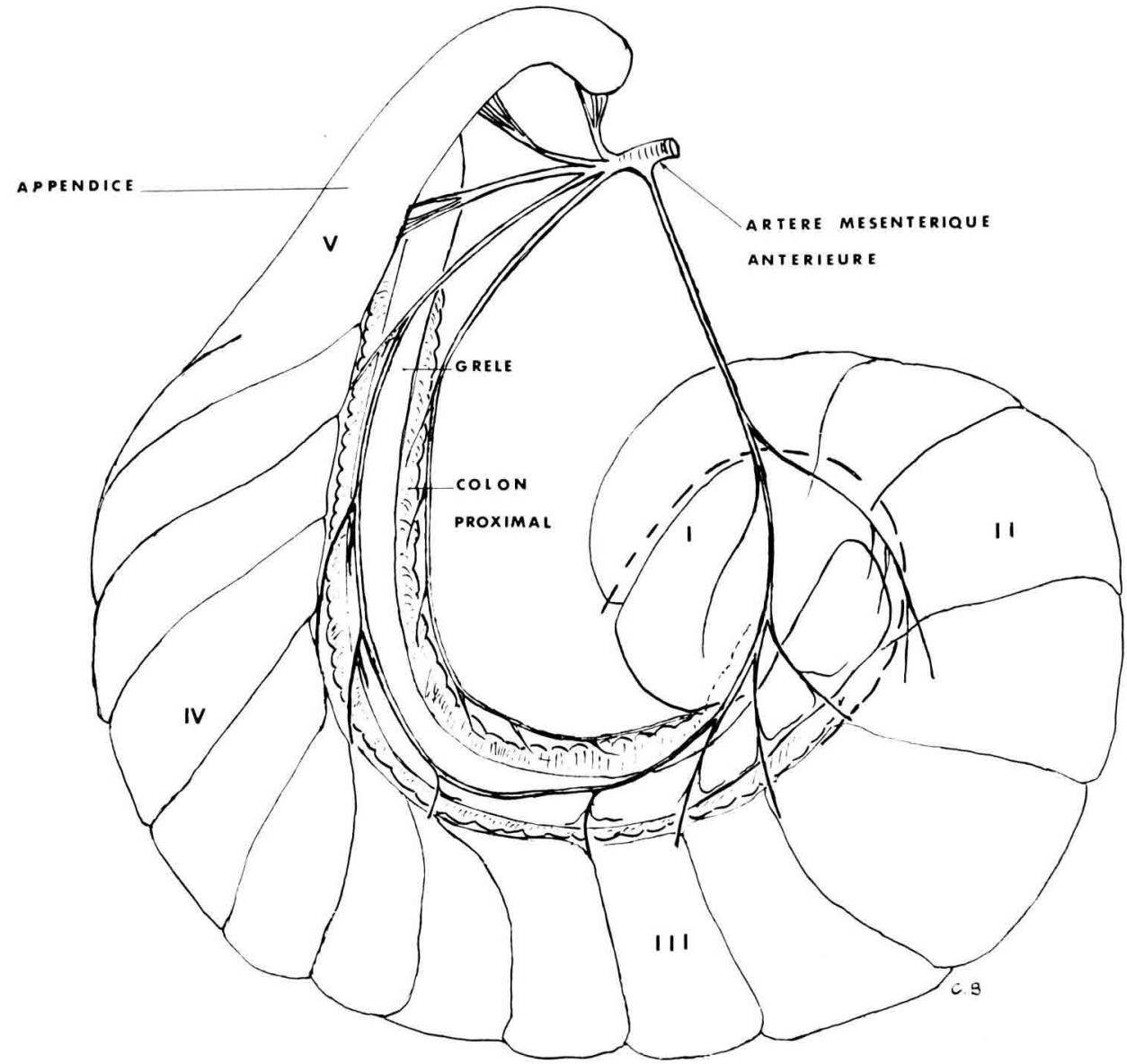

FIG. I. - Irrigation artérielle du cocum du Lapin

En trait discontinu la ligne d'incision pour l'ablation du cæcum

En chiffres romains, les lieux de prélèvement du contenu cæcal

\section{2. - Cecumectomie}

L'animal, à jeun depuis 12 heures reçoit par voie sous-cutanée $0,25 \mathrm{mg} / \mathrm{kg}$ de sulfate d'atrop:ne. Cette dose apparemment élevée est en fait bien tolérée à cause de la présence des atropi- 
nestérases et son action se prolonge au-delà du temps de l'intervention d'une durée de 45 minutes. Nous opérons dans les conditions d'asepsie chirurgicale.

Quinze minutes après atropine, le Lapin est anesthésié par injection veineuse de pentobarbital à raison de $30 \mathrm{mg} / \mathrm{kg}$ et fixé en décubitus dorsal. Le cæcum est extériorisé par une laparotomie abdominale médiane. Les vaisseaux mésentériques des anses cæcales sont ligaturés et le cæcum libéré de proche en proche.

Le contenu du cæcum est ramené vers la portion II par taxis manuel et un clamp est posé au voisinage de la valvule. Un deuxième clamp limitant permet de sectionner le cæcum et de l'éliminer. Les détritus végétaux sont retirés à l'aide de compresses imbibées de sérum physiologique et la muqueuse est largement irriguée sur champ stérile. Une suture en bourse perforante du type Cushing à la soie sertie no o permet de réaliser une fermeture hémostatique. Un deuxième surjet, enfouissant séro-séreux assure une parfaite étanchéité. On verse alors sur le péritoine 600 ooo U d'extencilline et l'on recoud la paroi à la soie. La peau est suturée par points au fil de lin. Les animaux reçoivent deux injections d'extencilline à 48 heures d'intervalle.

Dans ces conditions, la mortalité est faible (I sujet sur Io) et elle est toujours due à une ligature d'un vaisseau du grêle, le lapin ne tolérant pas l'anoxie intestinale localisée. Il nous est apparu primordial de conserver la valvule iléo-cæcale pour ne pas modifier tous les paramètres physiologiques du transit en même temps.

Nous avons ainsi procédé à l'ablation systématique du cæcum de cinquante lapins qui ont survécu plus de six mois. Une dizaine d'entre eux ont été maintenus en cages à métabolisme aux fins d'établir un bilan que nous avons comparé à celui des témoins. Les autres animaux ont été sacrifiés à des temps différents après l'intervention, allant de 15 jours à 6 mois pour déterminer le moment où pourrait se produire la restitution fonctionnelle de l'organe. Tous les animaux ont été autopsiés en fin d'expérience et des prélèvements systématiques réalisés en vue de l'analyse des lipides par portions de tube digestif.

\section{3. - Techniques d'incubation}

\section{a) In vitro.}

Le contenu cæcal est prélevé après anesthésie générale au pentobarbital sodique (30 $\mathrm{mg} / \mathrm{kg}$ ) et déposé dans un erlen à col rodé de $250 \mathrm{ml}$. On réalise 3 séries d'expériences : incubation de témoins, incubation de $50 \mathrm{~g}$ de contenu cæacal additionné de $0,5 \mathrm{ml}$ d'huile de lin ou d'huile de tournesol. Après homogénéisation, une partie aliquote est prélevée et traitée extemporanément pour l'analyse des lipides. Le reste est placé dans une étuve à $3^{\circ}$ pendant 24 heures sous atmosphère d'azote avant d'être analysé. La composition de ces huiles est donnée par le tableau I.

\section{b) In vivo.}

On sépare le cæcum de la valvule iléo-cæcale par deux sutures en bourse et on laisse l'organe en place muni de son irrigation et de son innervation. Un prélèvement témoin est réalisé au cours de l'intervention. On ajoute $5 \mathrm{ml}$ d'huile à l'aide d'une pipette au coeur de la masse digestive avant de procéder aux sutures.

L'animal est sacrifié au bout de six heures par saignée. On réalise de nouveaux prélèvements.

TABLEAU I

Pourcentages des divers constituants des huiles utilisées

\begin{tabular}{|c|c|c|c|c|c|c|c|c|}
\hline \multirow{2}{*}{ Huiles } & \multicolumn{3}{|c|}{ Classes de lipides } & \multicolumn{5}{|c|}{ Acides gras constituants } \\
\hline & $\begin{array}{l}\text { Trigly- } \\
\text { cérides }\end{array}$ & Stérols & $\begin{array}{l}\text { Mono- et } \\
\text { diglycérides }\end{array}$ & $\mathrm{C}_{16}$ & $\mathrm{C}_{18}$ & $\mathrm{C}_{18: 1}$ & $\mathrm{C}_{18: 2}$ & $\mathrm{C}_{18: 3}$ \\
\hline Tournesol & 88,2 & 4,8 & 6,7 & 8,5 & 4,2 & 25,2 & 62,2 & Traces \\
\hline Lin.... & 92,2 & 7,8 & 0 & 7,8 & 6,3 & 22,2 & 18,2 & 45,5 \\
\hline
\end{tabular}




\section{4. - Techniques d'analyse}

a) L'extraction des lipides à partir d'échantillons de 5 à $\mathrm{ro} g$ de contenu intestinal, de foie ou de graisse a été conduite selon la méthode de KAHANE (1963) : ébullition à reflux dans un mélange azéotropique benzène-éthanol (200/50. $v / v)$.

Les pigments végétaux sont éliminés en grande partie par cinq lavages à l'aide d'un mélange salin chloroforme-méthanol- $\mathrm{NaCl}$ 7,3 p. Ioo (Folch et al., 1957). 1944)

Les lipides du sang sont extraits à $40^{\circ}$ par le mélange méthylal méthanol (4/I $\left.v / v\right)$ (DELSAL,

b) La séparation des fractions se fait sur couche mince de silicagel de 0,25 mm d'épaisseur. Le solvant de migration est un mélange d'éther de pétrole-éther éthylique-acide acétique $180 / 20 / 2$ $v / v$ (BISERTE et al., I964). Dans ces conditions, les phospholipides et les galactoglycérolipides ne migrent pas. On évalue les pourcentages respectifs de chacune des classes en fonction des lipides neutres totaux par mesure des surfaces des taches après étalonnage. Dans ces conditions les mesures exprimées en pourcentage donnent une valeur indicative des modifications métaboliques, malgré les erreurs possibles dues à la migration simultanée de diverses classes chimiques telles que les esters de stérols (prédominants dans l'intestin du Lapin) et les hydrocarbures.

La fraction esters méthyliques est identifiée selon une technique que nous avons décrite antérieurement (BACQues et PERRET, I970).

c) Après saponification, les acides gras libérés par acidification du milieu sont recueillis dans l'heptane (LIPSKY et LANDOWNE, I963) puis méthylés par ébullition de 5 mn dans le mélange méthanol-trifluorure de bore à I 4 p. Ioo (MEtCalfe et Schmitz, I96I).

Les esters sont séparés par chromatographie en phase gazeuse sur colonnes imprégnées d'éthylène glycol succinate à $20 \mathrm{p}$. I 00 ou d'Apiézon, en programmation de température I6o à $24^{\circ}$ et détection par ionisation de flamme à l'aide d'un appareil " Perkin-Elmer type goo ".

\section{III. - RÉSULTATS}

\section{I. - Modifications anatomiques engendrées par l'ablation du cacum}

Chez le lapin nourri aux granulés, le cæcum ne se régénère pas.

Dans la plupart des cas nous observons une dilatation importante de la première partie du côlon proximal, dont le volume maximum est atteint en trois semaines. Ce phénomène a été décrit par HERNDON et Hove (I955).

Les animaux qui ne présentent pas de dilatation du côlon proximal ne survivent pas plus d'un mois et n'excrètent que des crottes molles. Chez les autres lapins, la dualité d'excrétion fécale se rétablit au bout d'un mois sans pour autant que réapparaisse la coprophagie.

\section{2. - Croissance et Bilan}

Après une chute rapide de poids pendant les deux premières semaines suivant l'ablation du cæcum, les lapins ont une courbe de croissance normale.

La dispersion des mesures de poids est plus grande chez les opérés que chez les témoins. Ceci est dû à ce qu'une partie de gain est liée à la dilatation du côlon proximal. On n'observe pas de troubles caractéristiques d'une avitaminose. Cependant, la fonte musculaire est sensible. Quand on fait le bilan quantitatif des gains de poids en fonction de la consommation de granulés au bout de 6 semaines, on constate que le cæcumectomisé utilise les granulés avec le même rendement que le témoin.

Le bilan hydrique est également modifié et l'on constate que la quantité quotidienne d'urine excrétée est en moyenne de $400 \mathrm{ml}$ contre $700 \mathrm{~m} 1$ pour le témoin. 


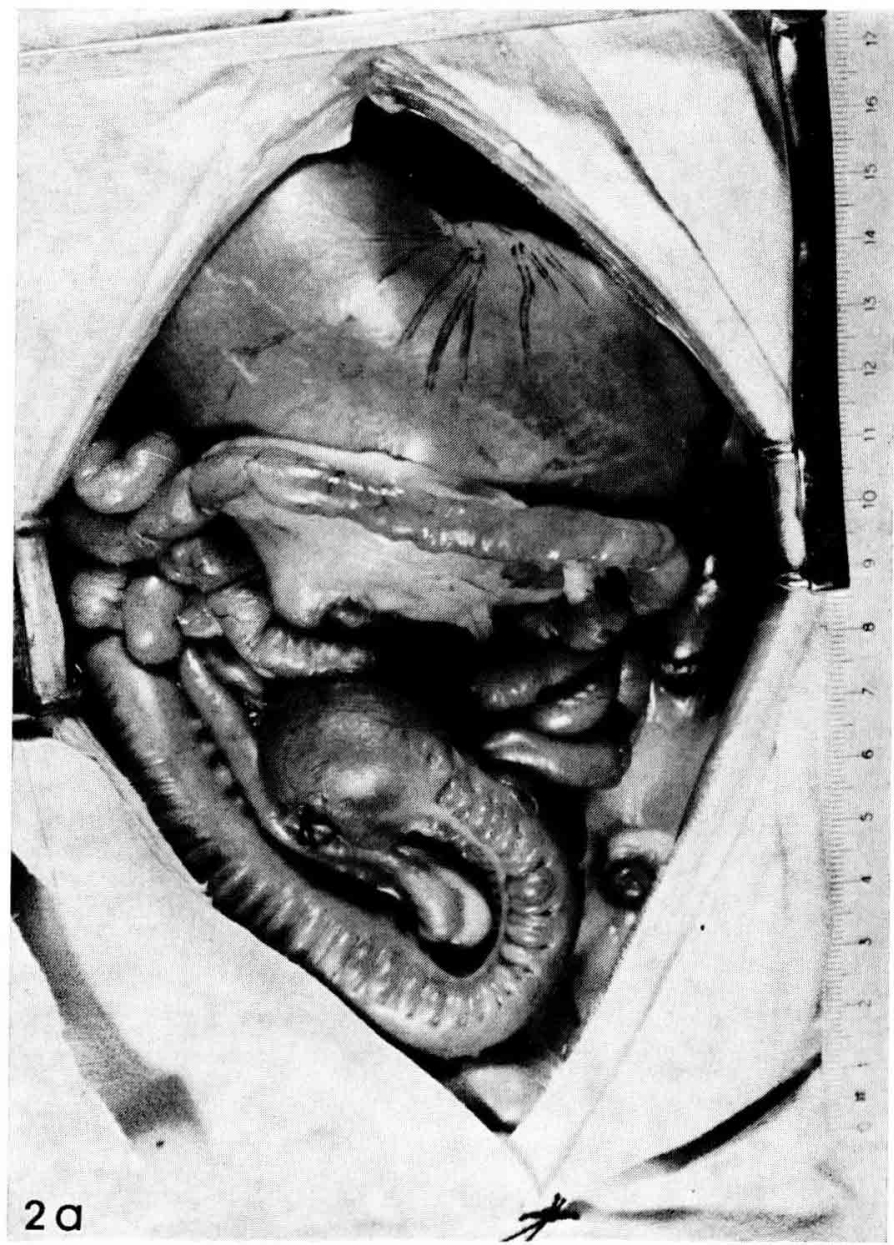

FIG. 2 a. - Viscères abdominaux du Lapin immédiatement après cacumectomie On remarquera l'importance du volume gastrique par rapport au côlon proximal

\section{3. - Étude des modifications fonctionnelles}

a) Répartition de l'eau dans le contenu intestinal.

Le contenu digestif est particulièrement riche en eau dans le grêle. Dans le cæcum, la déshydratation se fait progressivement de CI à CV mais elle reste partielle. Le côlon proximal absorbe une petite quantité d'eau et c'est dans le côlon distal que se fait de toute évidence la déshydratation du matériel fécal. Chez le lapin sans cæcum, le contenu de la valvule iléo-cæcale est riche en eau, de même pour le côlon proximal. Mais la réabsorption par le côlon distal, aboutit à la libération de crottes aussi peu hydratées que celles du témoin.

b) Répartition des lipides totaux dans le tube digestif.

La figure 3 nous montre que la teneur en graisse diminue progressivement depuis le grêle $(60 \mathrm{mg} / \mathrm{g}$ de poids sec) jusqu'au rectum. 


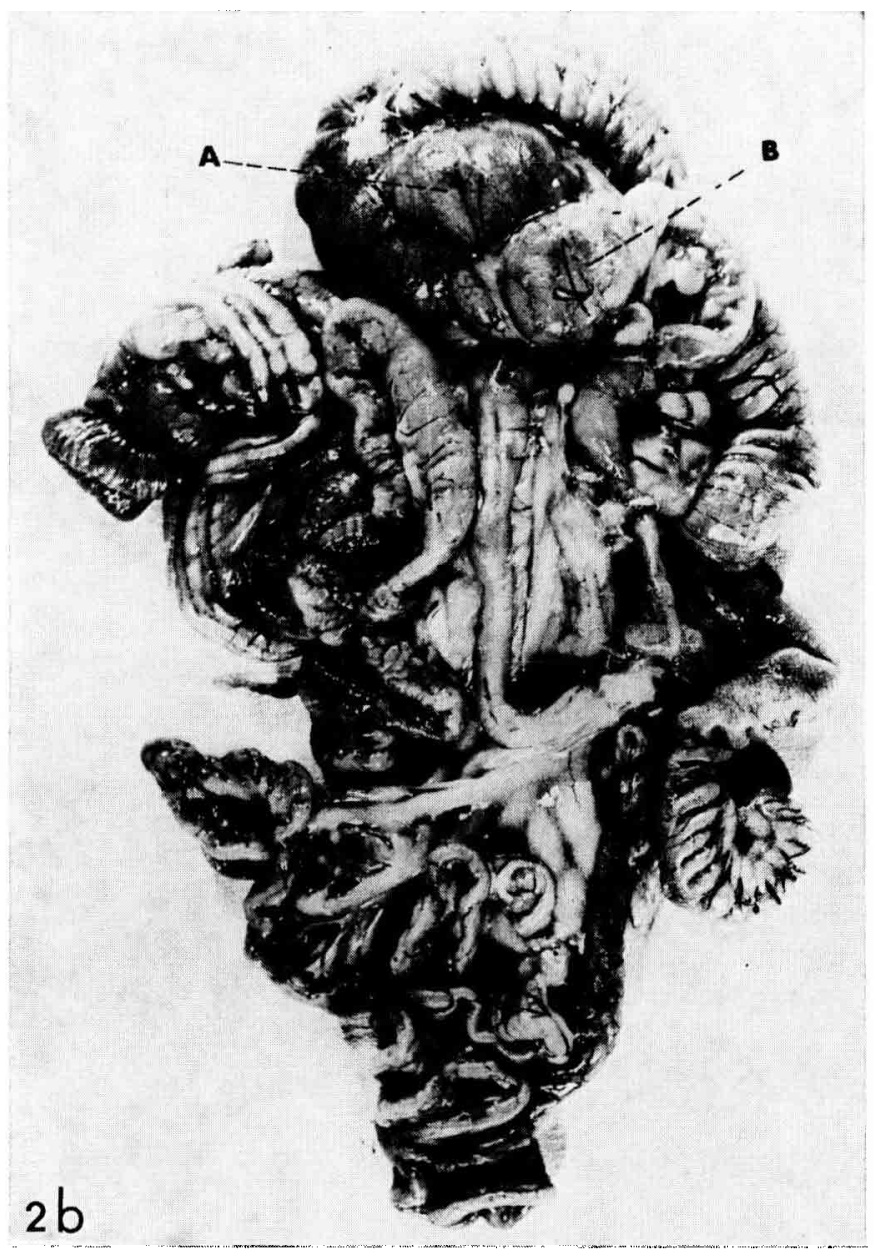

FIG. 2 b. - Hypertrophie du cólon proximal liée à la cacumectomie

A : côlon proximal

B : valvule iléo-cacale

Observation faite 2 mois après l'intervention

Dans le cæcum, la répartition des lipides est hétérogène car les teneurs trouvées dans la lumière de l'appendice sont deux fois plus élevées que celles de la portion médiane de l'organe.

Chez le lapin sans cæcum la quantité de lipides dans le grêle est inférieure à celle du témoin. Dans la région de la valvule iléo-cæcale le taux de lipides est abaissé de 60 p. I0o, alors que dans le côlon proximal on trouve des taux voisins de la normale. Ce phénomène pourrait être dû à l'accélération đu transit entre le grêle et le côlon à cause du faible calibre du tube digestif dans cette région pour une masse d'aliment ingérée toujours aussi importante. Dans tous les cas, le taux de lipides dans les crottes est le même pour le lapin témoin et l'animal opéré. En valeur absolue, les quantités moyennes de lipides totaux trouvées dans le tube digestif sont pour ro lapins témoins, de $\mathbf{2} 25, \mathrm{I} \mathrm{mg}$ dans le grêle, $633,3 \mathrm{mg}$ dans le cæcum, 95,4 mg dans le côlon proximal 
TABLEAU 2

Bilan métabolique de lapins témoins et sans cacum: moyennes effectuées après six semaines d'observation quotidienne

\begin{tabular}{|c|c|c|c|}
\hline & & $\begin{array}{l}\text { Témoins } \\
\text { (10 lapins) }\end{array}$ & $\begin{array}{l}\text { Sans cæcum } \\
\text { (10 lapins) }\end{array}$ \\
\hline \multirow{3}{*}{$\begin{array}{l}\text { Poids du lapin } \\
\text { en grammes }\end{array}$} & Départ & $3010 \pm 407$ & $2478 \pm 338$ \\
\hline & Après 6 semaines & 3610 土 485 & $2950 \pm 94$ \\
\hline & $\begin{array}{l}\text { Gain moyen } \\
\text { par semaine }\end{array}$ & $100,5 \pm 30$ & $78,7 \pm$ \\
\hline $\begin{array}{c}\text { Granulés ingérés } \\
(\mathrm{g} / 24 \mathrm{~h})\end{array}$ & $\begin{array}{l}\text { Poids frais } \\
\text { Poids sec }\end{array}$ & $\begin{array}{l}238 \pm 74 \\
215 \pm 60\end{array}$ & $\begin{array}{l}194 \pm 118 \\
175 \pm 70\end{array}$ \\
\hline $\begin{array}{l}\text { Crottes rejetées } \\
(\mathrm{g} / 24 \mathrm{~h})\end{array}$ & $\begin{array}{l}\text { Poids frais } \\
\text { Poids sec }\end{array}$ & $\begin{array}{rll}132 & \pm & 52 \\
93 & \pm & 23\end{array}$ & $\begin{aligned} 113 & \pm 61 \\
80 & \pm 25\end{aligned}$ \\
\hline $\begin{array}{l}\text { Différence : granulés } \\
\text { ingérés - crottes } \\
\text { rejetées }(\mathrm{g} / 24 \mathrm{~h})\end{array}$ & $\begin{array}{l}\text { Poids frais } \\
\text { Poids sec }\end{array}$ & $\begin{array}{l}106 \pm 84 \\
122 \pm 70\end{array}$ & $\begin{array}{l}81 \pm 70 \\
95 \pm 50\end{array}$ \\
\hline
\end{tabular}

\section{TABLEAU 3}

Étude comparée de la teneur en eau (mg/g de poids frais) du contenu des différents segments du tube digestif de lapins témoins et sans cacum

\begin{tabular}{|c|c|c|c|c|}
\hline & \multicolumn{2}{|c|}{ Témoin $(\mathrm{N}=11)$} & \multicolumn{2}{|c|}{ Sans cæcum $(N=5)$} \\
\hline & $m$ & $s$ & $m$ & $s$ \\
\hline Grêle & 868 & 69 & 882 & 50 \\
\hline C I & 773 & 29 & $806^{*}$ & 40 \\
\hline C II & 769 & 28 & & \\
\hline C III & 772 & 32 & & \\
\hline C IV & 759 & 27 & & \\
\hline $\mathrm{C} \mathrm{V}$ & 743 & 84 & & \\
\hline Côlon proximal & 743 & 5 & $815 * *$ & 61 \\
\hline Côlon distal & 679 & 5 & 727 & 158 \\
\hline Crottes & 570 & 112 & 572 & 99 \\
\hline
\end{tabular}


et $64, \mathrm{I} \mathrm{mg}$ dans le côlon distal. Ces chiffres, quoique susceptibles de grandes variations pour le contenu du grêle, sont, au contraire remarquablement stables pour le cæcum et le côlon, le réservoir cæcal jouant un rôle de régulateur dans le transit
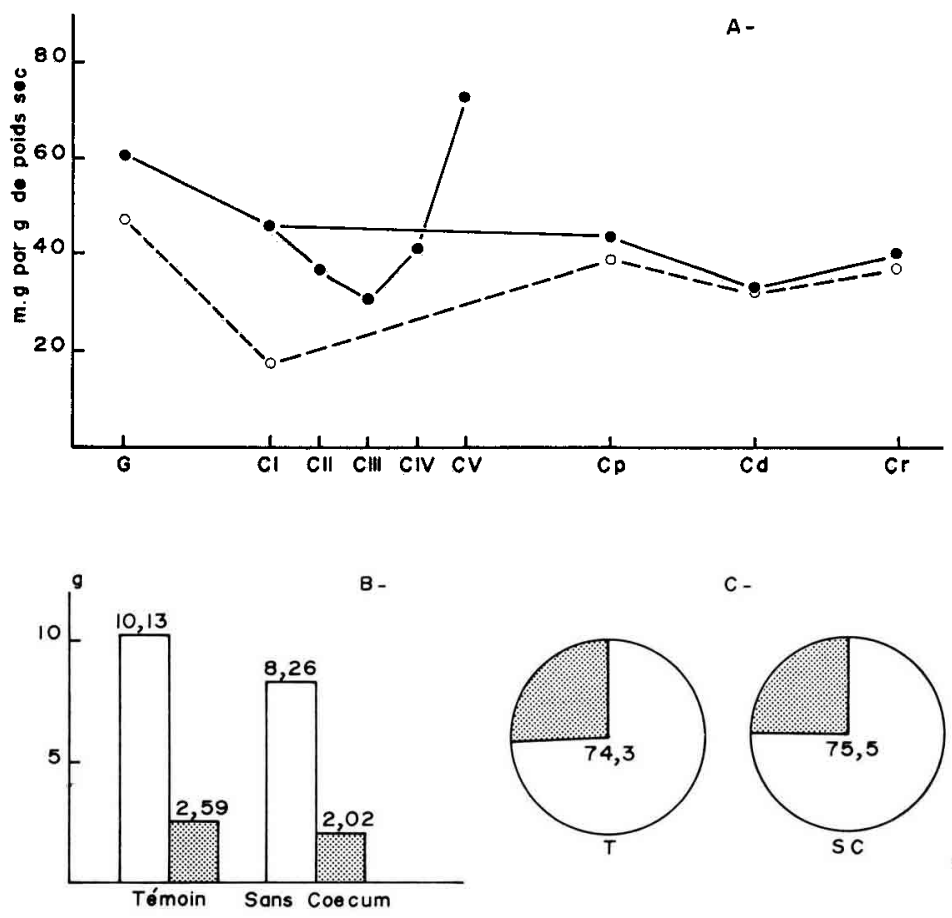

C -
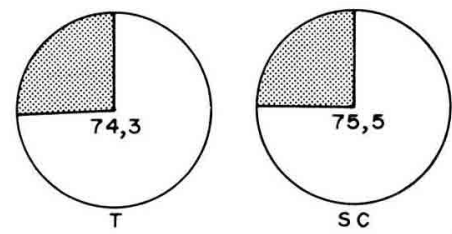

FIG. 3

A : teneur en lipides (mg/g de poids sec) du contenu intestinal des divers segments du tube digestif de lapins témoins - - et sans cæcum o-—o

$\mathrm{B}$ : digestion apparente des graisses chez les lapins témoins et sans cæcum. En blanc : graisses ingérées; en pointillés : graisses rejetées.

C : pourcentage des graisses apparemment digérées chez les lapins témoins et sans cæcum.

\section{TABLEAU 4}

Modifications de la répartition des classes de lipides provoquées par l'ablation du cacum chez le Lapin, exprimées en p. 100

\begin{tabular}{|c|c|c|c|c|c|}
\hline & \multirow{2}{*}{ Granulés } & \multicolumn{2}{|c|}{ Côlon proximal } & \multicolumn{2}{|c|}{ Crottes: } \\
\hline & & Témoin & Cæcumectomisé & Témoin & Cæcumectomise \\
\hline Stérols estérifiés $\ldots \ldots \ldots \ldots$ & 48 & 19,8 & 12,9 & 53,2 & 29,9 \\
\hline gras $\ldots \ldots \ldots \ldots \ldots \ldots$ & 0 & 8,9 & 9,0 & 10,4 & 15,3 \\
\hline Triglycérides $\ldots \ldots \ldots \ldots$ & 24 & 24,3 & 29,2 & 12,4 & 14,6 \\
\hline Mono- et diglycérides & 7,4 & 7,4 & 3,6 & 8,5 & 7,7 \\
\hline Acides gras libres ........ & 13,6 & 27,1 & 37,5 & 8,9 & 23,8 \\
\hline Stérols $\ldots \ldots \ldots \ldots \ldots \ldots$ & 7 & 12,5 & 7,8 & 6,7 & 8,3 \\
\hline
\end{tabular}


des graisses. Il semble qu'une absorption ait lieu au niveau du côlon proximal ou du côlon distal. Chez le cæecumectomisé, les lipides du côlon proximal sont moins abondants $(80,8 \mathrm{mg})$, et dans le côlon distal, on en trouve une quantité équivalente, ce qui pose le problème de l'absorption des lipides par le côlon en fonction de leur nature

\section{c) Modifications de la composition des lipides engendrées par l'ablation du caecum.}

c $\left._{1}\right)$ Modifications de la répartition des lipides par classes.

Parmi l'ensemble des mesures effectuées par chromatographie en couche mince des différentes classes de lipides, les plus caractéristiques relèvent de l'analyse du contenu du côlon proximal et des crottes.

Dans le côlon proximal on trouve sensiblement moins d'esters de stérols que chez le témoin, et, à l'opposé, beaucoup plus d'acides gras libres. Dans les crottes, la répartition est semblable dans les deux cas, à l'exception des stérols estérifiés deux fois moins abondants chez l'animal opéré et des acides gras libres en quantité trois fois plus grande.

Dans le grêle, les proportions relatives des acides gras étudiés sont les mêmes pour le témoin et l'animal opéré. A ce niveau, on note une chute importante du taux de $\mathrm{C}_{18: 1}$ par rapport au contenu des granulés. Le devenir de cet acide devra être précisé par l'emploi de corps radioactifs. Par contre, au niveau de la valvule iléocæcale, on constate une baisse de 52 p. Ioo de l'acide palmitique. Cet écart est maintenu dans le côlon proximal et dans les crottes. L'ablation du cæcum s'accompagne également d'une élévation du taux des acides insaturés en $\mathrm{C}_{18: 1,{ }_{2}}$ et ${ }_{3}$ dans le côlon proximal. Dans les crottes, le taux du $\mathrm{C}_{18: 3}$ reste élevé.

$\mathrm{c}_{2}$ ) Modifications apportées par la cacumectomie sur les acides gras du plasma, du foie et des graisses de réserve.

Compte tenu des échanges permanents entre les acides gras du sang et du tissu adipeux (MOORE et WILLIAMS, I966; MoORE et WILLIAMS, I968) il est nécessaire de contrôler les effets de la cæcumectomie sur la nature des lipides circulants ou mis en réserve. La graisse mésentérique naturellement riche en acides insaturés (CLEMENT et MEARA, I95I) ne change pas de composition en acides gras après l'intervention. Par contre, les lipides du foie s'enrichissent en acides insaturés $\mathrm{C}_{18: 2}$ et $\mathrm{C}_{18: 3}$ de façon significative. La teneur en $\mathrm{C}_{16}$ est abaissée de $35 \mathrm{p}$. Ioo. Dans le sang artériel, $\mathrm{C}_{18 ; 1}$ et $\mathrm{C}_{18 ; 3}$ sont plus abondants.

\section{4. - Incubations en présence d'un substrat huileux}

I. Étude in vitro.

a) Après incubation de 12 heures.

On observe une hydrolyse marquée des triglycérides (22,8 à 56,8 p. Ioo) semblable pour les deux huiles. Des acides gras sont libérés. L'hydrolyse de l'huile de lin est toujours plus complète que l'huile de tournesol. Les esters des stérols se présentent toujours en fort pourcentage, pour les témoins comme pour les essais. Le taux des esters méthyliques varie dans le même sens que celui des acides gras libres. 
焉

5

:

\&ั 눈

in

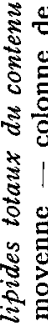

\&

s.

केष

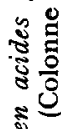

है

\begin{tabular}{|c|c|c|}
\hline \multirow{4}{*}{ 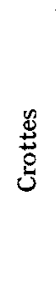 } & \multirow{2}{*}{ 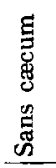 } & 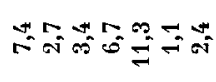 \\
\hline & & 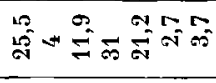 \\
\hline & \multirow{2}{*}{ 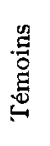 } & $\begin{array}{c}0 \\
0 \\
0\end{array}$ \\
\hline & & 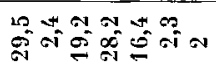 \\
\hline \multirow[b]{2}{*}{ 焉 } & 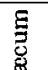 & 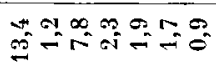 \\
\hline & ڤั๊ & 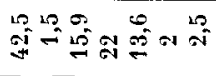 \\
\hline
\end{tabular}

$$
\text { 过 }
$$

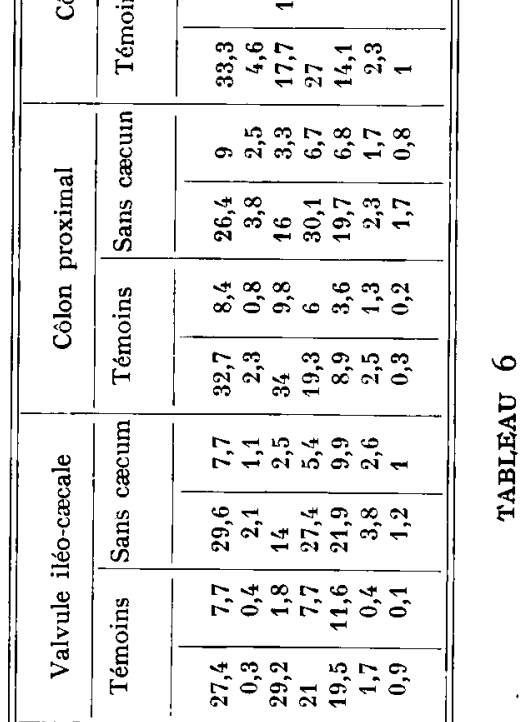

\begin{tabular}{|c|c|c|}
\hline \multirow{2}{*}{\multicolumn{2}{|c|}{ 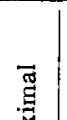 }} & $\sigma$ नी \\
\hline & & \\
\hline
\end{tabular}

\begin{tabular}{|c|c|c|}
\hline \multirow{4}{*}{ 离 } & \multirow{2}{*}{ 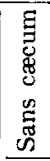 } & 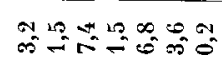 \\
\hline & & 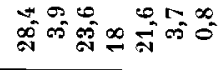 \\
\hline & \multirow{2}{*}{ 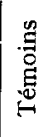 } & $\begin{array}{l}\infty \\
0 \\
0\end{array}$ \\
\hline & & 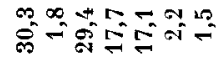 \\
\hline \multirow{2}{*}{ 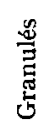 } & $n$ & $\begin{array}{ll}\mathbb{A} & : 0 \\
0 & 0\end{array}$ \\
\hline & $\Sigma$ & 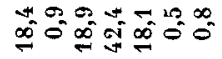 \\
\hline \multicolumn{2}{|c|}{ 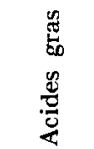 } & "ేّ \\
\hline
\end{tabular}

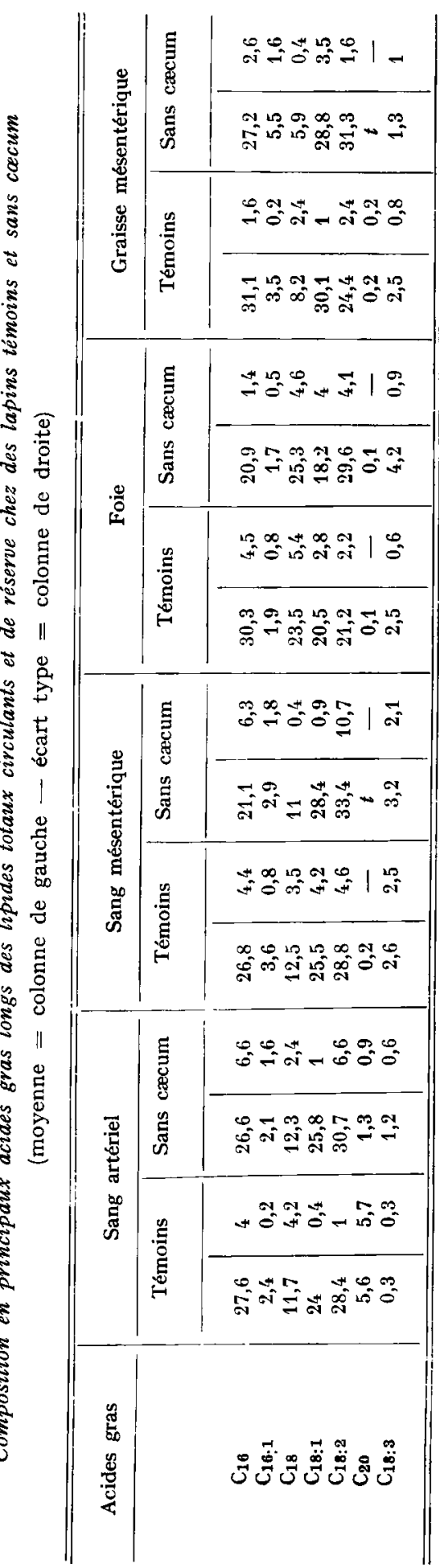



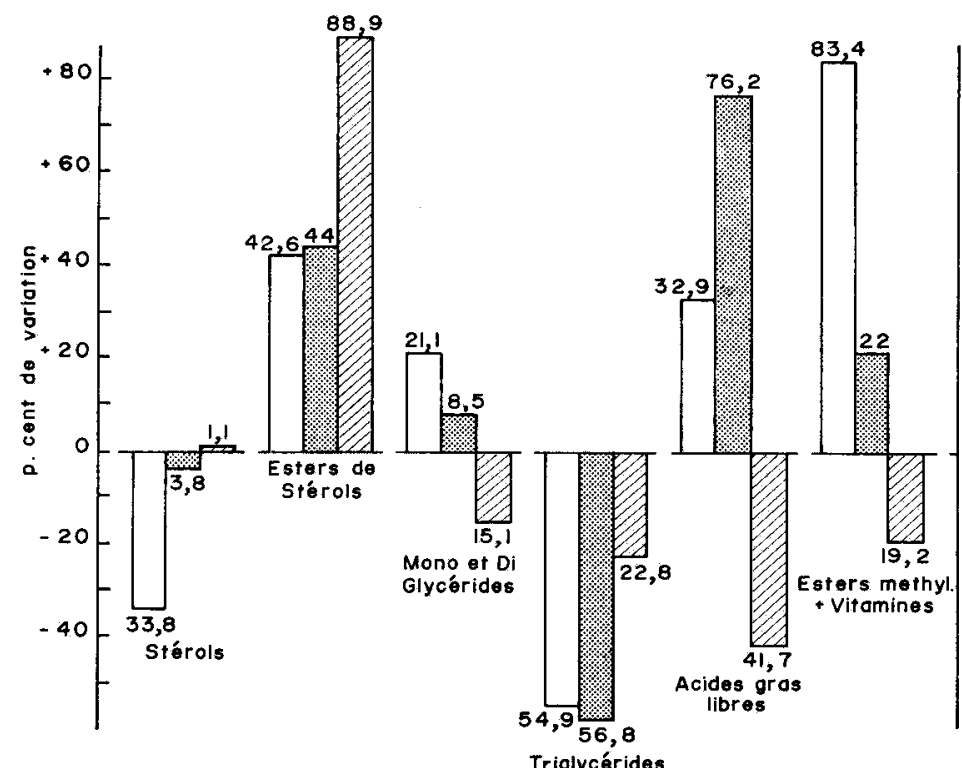

FIG. 4. - Variations de la composition lipidique du milieu (exprimée en p. Ioo) après l'incubation (à $37^{\circ} \mathrm{C}$ pendant 24 heures et sous azote) du contenu coecal de lapins en présence de divers substrats

$$
\begin{aligned}
& \text { Colonnes blanches }=\text { huile de tournesol } \\
& \text { Colonnes pointillées }=\text { huile de lin } \\
& \text { Colonnes hachurées }=\text { pas de substrat }
\end{aligned}
$$

\section{b) Cette hydrolyse s'accompagne d'une hydrogénation.}

D'une façon générale, après incubation, le taux d'acide stéarique augmente dans des limites significatives $(6,3$ à $40,3 \mathrm{p}$. IOO). Au contraire, celui des acides olèique et linolénique s'abaisse de I4,6 $\mathrm{p}$. Ioo pour $\mathrm{C}_{18: 1}$ et de $60,3 \mathrm{p}$. Ioo pour $\mathrm{C}_{18: 3}$.

L'acide linoléique n'est saturé que très partiellement. Son hydrogénation n'est perceptible réellement que dans les expériences où l'acide a été introduit seul dans le milieu. Il semble bien qu'il y ait une préférence des réactions de saturation pour les acides mono ou tri insaturés.

\section{2. Étude in vivo.}

Les phénomènes observés in vitro sont accentués in vivo, en particulier, les réactions d'hydrolyse des triglycérides et la synthèse d'esters de stérols. Ceux-ci, récupérés après migration par chromatographie en couche mince puis saponifiés, fournissent essentiellement des acides gras insaturés en $\mathrm{C}_{1 \xi_{1}}$ et $\mathrm{C}_{1 \% 3}$.

La quantité de lipides totaux retrouvés en fin d'expérience montre qu'au niveau de cet organe leur absorption est pratiquement nulle. 


\section{IV. - DISCUSSION}

L'exposé des observations réalisées sur animal normal ou cæcumectomisé nous conduit à l'élaboration de questions nouvelles :

Alors que la cæcumectomie est toujours fatale chez le rat à longue échéance par avitaminose $B$, elle ne l'est pas chez le Lapin où cet organe représente cependant un réservoir bactérien très important. La coprophagie supprimée par la cæcumectomie, ne peut pas s'exprimer par un besoin alimentaire en un produit de la digestion. Yoshida et al. (I968) ainsi que REDDY et al. (1965) ont observé le même phénomène sur lapin germ free. Tout laisse penser que cet habitus du Lapin est lié à la présence dans les crottes d'une substance odorante d'origine bactérienne, analogue aux aldéhydes gras synthétisés par les bactéries du rumen et isolées par KATZ et KEENEY (rg62) chez le Mouton.

Les prélèvements systématiques le long du tube digestif nous montrent bien que l'homogénéité physique du contenu cæcal mis en évidence par 1'utilisation d'oxyde de chrome (YoshIHARA et KaNDATSU, I960) ne correspond pas à une réalité sur le plan biochimique. D'autre part, on peut reconnaître au côlon distal une fonction particulière de régulation dans l'absorption-sécrétion des graisses car les crottes émises par tous les animaux sont plus riches en esters que le contenu du côlon proximal.

Il se passe dans le cæcum des phénomènes analogues à ceux qui sont observées dans la panse des ruminants : hydrolyse des lipides et hydrogénation. La première réaction, dans le rumen, ne peut être attribuée à la lipase pancréatique qui sera libérée beaucoup plus loin, mais à l'action bactérienne (BATH et HILL, 1967; GARTON et al., I96I ; HAWKE et ROBERTSON, I964; REISER, I95I).

La saturation des acides se fait par un processus analogue : SHORLAND et al. 1955, 1957) ont montré que l'acide linolénique est hydrogéné préférentiellement aux autres acides et que les acides à doubles liaisons conjuguées sont peu modifiés. Cette préférence bactérienne pour le $\mathrm{C}_{18: 3}$ se retrouve donc chez le Lapin. Par contre, l'estérification des stérols ne semble pas avoir retenu l'attention des chercheurs en matière de digestion dans le rumen. Ceci pourrait être dû à une alimentation généralement différente de celle de nos lapins.

Le fait qu'il y ait davantage d'acides gras libres dans le côlon proximal et dans les crottes de l'animal opéré rappelle les phénomènes observés habituellement chez 1'Homme, le Chien, le Rat en insuffisance pancréatique partielle ou totale. De même pour les esters de stérols. Les lipases d'origine bactérienne ou provenant de la desquamation de la muqueuse cæcale jouent probablement un rôle majeur. C'est ce que nous tentons de déterminer actuellement par l'exclusion systématique de la sécrétion exocrine du pancréas. L'estérification préférentielle des stérols par les estérases pancréatiques reste à démontrer au niveau du côlon de l'animal opéré.

L'administration de fortes doses de cholestérol à des lapins par voie digestive provoque une mobilisation importante des acides gras des tissus de réserve et une excrétion accrue d'esters de cholestérol (PoPJAK I946). Compte tenu des différences observées entre les teneurs de ces esters dans le côlon proximal et les crottes, nous émettons l'hypothèse de la localisation au côlon distal de cet te excrétion. 


\section{V. - CONCLUSIONS}

La cæcumectomie chez le Lapin est compatible avec une survie prolongée. Elle n'intervient pas dans la croissance de l'animal ou le bilan métabolique global des lipides. Elle permet de mettre en évidence le rôle du cæcum dans l'hydrolyse des triglycérides, et la saturation des acides en $\mathrm{C}_{18 \mathrm{x}},{ }_{2}$ et ${ }_{3}$.

L'estérification de stérols pourrait être rapportée à l'activité lipasique du pancréas et des bactéries. L'ensemble des faits exposés ouvre une voie nouvelle de recherche.

Reçu pour publication en octobre 1970.

\section{SUMMARY}

\section{CECUMECTOMY INDUCED ALTERATIONS IN THE DISTRIBUTION AND NATURE OF L,IPID CONSTITUENTS IN THE DIGESTIVE FLUID OF THE RABBIT}

Very little is known about the digestion of lipids by the rabbit; our study purports to gain some insight into the distribution of lipids in the digestive tract and the changes induced by cecumectomy.

Cecumectomy was performed on 50 rabbits. 20 rabbits were used as controls. The lipolytic effect of the cecal contents was evidenced by means of in vitro and in vivo incubation techniques described in the test.

Our results were as fellows:

I. Growth rate was irrespective of cecumectomy. The rabbits lived for more than a year after the operation. Coprophagy disappeared and the pellet intake was smaller.

$2 a$. The digestive fluid of the cecumectomized animal contained more water, which was absorbed at the lower large intestine level, allowing normal feces.

$2 b$. Total daily lipid excretion was higher after the operation. Its composition was mainly free fatty acids.

$2 c$. The fatty acids occuring during sterol esterification (viz. $C_{18}: 1,2,3$ ) remain unsaturated. Hydrogenation depends upon the presence of bacteria.

These results further substantiate our investigation program using methods of systematic lock-out of organs or secretions.

\section{RÉFÉRENCES BIBLIOGRAPHIQUES}

Bacoues Cl., Perret J.-P., 1970. Mise en évidence de la présence d'esters méthyliques d'acides gras dans le tractus digestif du lapin en alimentation contrôlée. C. R. Acad. Sci, 270, r8o7-18ro.

Barcroft J., McAnally R. A., Phillipson A. T., I944. Absorption of volatile fatty acids from the alimentary tract of the sheep and other animals. J. Exptl. Biol., 20, 120-129.

Bath I. H., HiLl K. J., 1967. The lipolysis and hydrogenation of lipids in the digestive tract of the sheep. J. agric. Sci. Camb., 68, I39-I48.

Biserte C., Sezille G., Bertrand M., Jaillard J., ig64. Exploration lipidique au cours du diabète. Ann. Biol. Clin., 22, 86r-897.

Bloom B., Chaikoff I. L., Reinhardt W. O., r95I. Intestinal lymph as the pathway for transport of absorbed fatty acids of different chain length. Amer. J. Physiol., 166, 45I-459.

Bonnafous R., Raynaud P., I968. Mise en évidence d'une activité Jysante du côlon proximal sur les microorganismes du tube digestif du lapin. Arch. Sci. Physiol., 22, 57-64. 
Clement G., Meara M. L., I95I. Components acids of the perinephric and interscapular fats of a rabbit. Biochem. J., 49, 561-57I.

Cools A., Jeuniaux Ch., I96r. Fermentation de la cellulose et absorption d'acides gras volatils au niveau du cæcum du Lapin. Arch. Internat. Physiol. Biochim., 69, I-8.

Cook R. P., Thомson R. O., I95I. Cholesterol metabolism. III. Cholesterol metabolism in the guinea pig and rabbit. Biochem. J., 49, 72-77.

Cook R. P., Thomson R. O,, r95I. Absorption of fat and cholesterol in the rat, guinea pig and rabbit. Quart. J. Exp. Physiol., 36, 61-74.

Coullaud D., Lévè Que J., 1966. Contribution à l'étude de la motricité intestinale chez le Lapin. J. Physiol., 58, 502.

Delsal. J.-L., I944. Nouveau procédé d'extraction des lipides du sérum par le méthylal. Applications aux microdosages du cholestérol total, des phospho-amino-lipides et des protéinés. Bull. Soc. Chim. Biol., 26, 99-101.

Ducluzeatu R., r969 Infiuence de l'espèce zoologique sur la microflore du tractus gastro-intestinal. Rev. immunol., 33, 345-383.

ERwin J., Bloch K., I964. Biosynthesis of insatured fatty acids in micro-organisms. Science, 143, roo6-I012.

Folch J., Lees M., Sloane-Stanley G. H., I957. A simple method for the isolation and purification of total lipids from animal tissues. J. Biol. Chem., 226, 497-499.

Garton G. A., Lough A. K., Viogue E., I96r. Glyceride hydrolysis and glycerol fermentation by sheep rumen contents. J. Gen. Microbiol, 25, 215*225.

Hawke J. C., Robertson J. A., I964. Studies on rumen metabolism. II. In vivo hydrolysis and hydrogenation of lipid. J. Sci. Fd. Agric., 15, 283-289.

Herndon J. F., Hove E. L., I955. Surgical removal of the cecum and its effect on digestion and growth in rabbits. J. Nutr., 57, 261-272.

KAhane E., I963. Extraction des lipides par un procédé azéotropique. C. R. Acad. Sci., 257, r966-ı968.

Kametaka M., I967. Studies on the digestion in the rabbit. Part XI. Some physicochemical properties of digesta in the small intestinal tract of the rabbit fed on a pellet diet. Agr. Biol. Chem., 31, 6I6-624.

KATZ I., KEENEY M., I962. Characterization of the octadecenoique acids in rumen digesta and rumen bacteria. Biochem. Biophys. Acta, 84, 128-132.

Kotani M., Seiki K., Yamashita A., Takashima A., Nakagawa T., Hori I., ig67. Concentration and composition of fatty acids of thoracic duct lymph lipids from rabbits. Amer. $J$. Physiol., 213, $253-238$.

LipsKy S. R., LAndowne R. A., I963. The identification of fatty acids by gaz chromatography. Enzymol. U. S. A., 6, 5I3-537.

Metcalfe L. D., Schmitz A. A., Ig6I. The rapid preparation of fatty acid esters for gaz chromatographic analysis. Anal. Chem., 33, 363-364.

Moore J. H., Williams D. L., I966. The effect of diet on the composition of the triglycerides and unesterified fatty acids isolated from the plasma, liver and adipose tissues of rabbits. $B r . J . N u t r$., 20,79 .

Moore J. H., Williams D. L., I968. The effect of diet on the composition and positional distribution of the fatty acids in the triglycerides obtained from the adipose tissues of rabbits. Brit. $J . N u t r ., 22$, $473-482$.

PoPJAK G., 1946. The effect of feeding cholesterol without fat on the plasma lipids of the rabbit. The role of cholesterol in fat metabolism. Biochem., 40, 608-62 I.

Reddy B.S., Pleasants J. R., Zimmerman D. R., Wostman B. S, I965. Iron and copper utilization in rabbits as affected by diet and germ free status. J. Nutr., 87, I89-196.

Reiser R., I95I. Hydrogenation of polyunsaturated fatty acids by the ruminant. Fedn. Proc. Fedn Amer. Soc. Exp. Biol., 10, 236.

ScheuerbrandT G., Block K., Ig62. Unsaturated fatty acids in microorganisms. J. Biol. Chem., 237, 2064-2068.

Shorland F. R., WeEninck R. O., Johns A. T., I955. Effect of the rumen on dietary fats. Nature, 175, II29.

Shorland F. R., Weeninck R. O., Johns A. T., McDonald I. R. C., r957. The effect of sheep rumen contents on unsaturated fatty acids. Biochem. $J ., 67,328$.

Viallard V., Raynaud P., 1968. Présence d'un agent bactériolytique dans le contenu de l'estomac du Lapin. J. Physiol., 60,323.

Yoshida T., Pleasants J. R., Ready B. S., Wostman B. S., x968. Efficiency of digestion in germ free and conventional rabbits. Brit. J. Nutr., 22, 723-737.

Yoshihara I., Kandatsu M., I960. Studies on cecum digestion. IV. On the movement of cecal contents in the rabbit. Bull. Agr. Chem. Soc. Japan, 24, 543-547. 